\title{
Positron Annihilation Studies of the Electronic Structure and Fermiology of High- $T_{c}$ Superconductors*
}

\author{
L. C. Smedskjaer \\ Materials Science Division, Argonne National Laboratory, Argonne, IL 60439
}

\author{
A. Bansil \\ Physics Department, Northeastern University, Boston, Massachusetts 02115 \\ Z. Naturforsch. 48a, 398-405 (1993); received January 25, 1992
}

\begin{abstract}
We discuss the application of the positron annihilation angular correlation (ACAR) spectroscopy for investigating the electronic structure and Fermiology of high- $T_{\mathrm{c}}$ superconductors, with focus on the $\mathrm{YBa}_{2} \mathrm{Cu}_{3} \mathrm{O}_{7}$ system where most of the experimental and theoretical work has to date been concentrated. Detailed comparisons between the measured 2D-ACAR positron spectra and the corresponding band theory predictions show a remarkable agreement (for the normal state), indicating that the electronic structure and Fermi surface of this material is described reasonably by the conventional picture.
\end{abstract}

Key words: Positron annihilation; Angular correlation of annihilation radiation (ACAR); Electron momentum density; High- $T_{\mathrm{c}}$ superconductors.

\section{Introduction}

Since the discovery of superconductivity in 1911 [1], it was realized that this phenomenon, manifesting ideal diamagnetism and zero resistance, represents a unique state of matter. A fundamental understanding of superconductivity began to emerge much later by the BCS theory [2]. Over the years many superconductors were discovered, but the highest transition temperature until 1987 was approximately $23 \mathrm{~K}$, when Bednorz and Müller initiated the discovery of a whole new class of materials with much higher $T_{\mathrm{c}}$ 's [3]. In addition to their technological potential, the new superconductors immediately raised fundamental questions concerning the nature and mechanism of superconductivity itself, and spawned new theoretical models, such as the resonance valence bond (RVB), marginal and modified Fermi-liquid scenarios, spin bags, Hubbard-based models, among others. A great deal of effort has also been devoted to the conventional band theory model based on the local density approximation (LDA) [4].

The question of which, if any, of these models describes the Fermi gas in the new materials adequately, must of course eventually be decided by experiment.

* Presented at the Sagamore X Conference on Charge, Spin and Momentum Densities, Konstanz, Fed. Rep. of Germany, September 1-7, 1991.

Reprint requests to Dr. L. C. Smedskjaer, Materials Science Division, Argonne National Laboratory, Argonne, IL 60439, USA.
Here, as stressed at the recent Argonne Fermiology Workshop [4], the $\boldsymbol{k}$-resolved spectroscopies, which probe individual $\boldsymbol{k}$-states or small groups of such states, may be particularly useful in identifying the characteristic signatures of the spectral properties at and near the Fermi energy. For these reasons, the recent focus has been on the positron annihilation angular correlation (ACAR), angle-resolved photoemission (ARPES), and the de Haas-van Alphen (dHvA) techniques. Although little Compton scattering work has to date been reported on the high- $T_{\mathrm{c}}$ 's, the technique offers unique possibilities in exploring the Fermiology of compounds. It is noteworthy that the LDA-based band theory model is the only model where detailed comparisons between theoretical predictions and the spectroscopic intensities have to date been possible.

The purpose of this article is to discuss the contribution of $2 \mathrm{D}$-ACAR positron technique towards the understanding of the electronic structure of the new superconductors. In this connection, a few historical remarks concerning how the positron spectroscopy has emerged as a modern tool for investigating electronic structures may be appropriate. The first angular correlation experiment to our knowledge was reported by Beringer and Montgomery in 1942 [5], followed by one of the earliest theoretical decriptions of the positron annihilation in metals by De Benedetti et al. [6]. The pioneering work aimed at using positron annihilation as a probe of the electronic structure was 
initiated in the late 50's by De Benedetti [7], Stewart [8], and Berko [9]. The article by Berko and Plaskett appears to be the first study of a metal single crystal and furthermore addresses the question of a detailed comparison between theory and experiment. Over the years, the experimental techniques have improved dramatically, culminating with the coming on line of the $2 \mathrm{D}$ positron cameras [10-12]. The theoretical methodology for interpreting and analyzing the positron spectra within the band theory framework has also made great strides, including the treatment of complex materials, spurred in part by the availability of ever more powerful computers. It was natural then that as the questions concerning the Fermiology of the new superconductors arose with their discovery, positron annihilation was among the first spectroscopies to be brought to bear on the problem.

An outline of this article is as follows. The introductory remarks are followed in Sect. II by some comments comparing Compton scattering and positron annihilation techniques. Section III gives an overview of the existing 2D-ACAR work in the high- $T_{\mathrm{c}}$ 's. Section IV focuses on the classic $\mathrm{YBa}_{2} \mathrm{Cu}_{3} \mathrm{O}_{7-x}$ compounds, where most of the work has been concentrated. A few concluding remarks are made in Section V.

\section{Positron Annihilation vs. Compton Scattering}

The spectral quantity involved in a positron annihilation experiment is the electron-positron momentum density, $\varrho_{2 \gamma}(p)$, given in the independent-particle model by the expression [13]

$\varrho_{2 \gamma}(\boldsymbol{p})=$ const $\sum_{j}\left|\int \exp (-i \boldsymbol{p} \cdot \boldsymbol{r}) \psi_{+}(\boldsymbol{r}) \psi_{j}(\boldsymbol{r}) \mathrm{d} \boldsymbol{r}\right|^{2}$,

where $\psi_{+}(\boldsymbol{r})$ is the positron wave function and $\psi_{j}(\boldsymbol{r})$ denotes electron wave functions for the $j$-th band, and the summation is extended to all occupied electron states. The Compton experiment, on the other hand, is related to the electron momentum density, $\varrho(\boldsymbol{p})$, defined by removing the positron wave function from (1) as [14]

$$
\varrho(\boldsymbol{p})=\sum_{j}\left|\int \exp (-i \boldsymbol{p} \cdot \boldsymbol{r}) \psi_{j}(\boldsymbol{r}) \mathrm{d} \boldsymbol{r}\right|^{2} .
$$

A positron annihilation angular correlation (ACAR) experiment, aside from resolution effects, measures a projection of $\varrho_{2 \gamma}(\boldsymbol{p})$. In $2 \mathrm{D}-\mathrm{ACAR}$, a projection along one dimension is involved, i.e.

$$
M\left(p_{x}, p_{y}\right)=\int \varrho_{2 \gamma}(\boldsymbol{p}) \mathrm{d} p_{z},
$$

where the integration is along the axis of the positron camera. The Compton scattering experiment, however, usually involves a $1 \mathrm{D}$-projection over two dimensions (much like the older $1 \mathrm{D}-\mathrm{ACAR})$, of $\varrho(p)$

$$
J\left(p_{z}\right)=\iint \varrho(p) \mathrm{d} p_{x} \mathrm{~d} p_{y} .
$$

The application of (1)-(4) to a specific system requires the choice of a particular band-structure methodology. We refer to the literature for the formalism appropriate for the many-atom-per-unit-cell case that is required for treating the high- $T_{\mathrm{c}}$ 's [15-22]. Note also that (1) neglects electron-positron correlation effects; for a discussion of methods of including such effects see $[20,23]$.

In comparing Compton scattering and positron annihilation, we note that the interpretation of positron spectroscopy is complicated by the presence of positron spatial distribution effects, and by the theoretical uncertainties inherent in the treatment of electron-positron correlations. The positron, however, is repelled by the ionic cores, and therefore the annihilation process preferentially samples the valence rather than the core electrons. Compton scattering, in contrast, involves all electrons, and thus yields a relatively smaller signal from the valence states. We should keep in mind, nevertheless, that in the 2D-ACAR experiment only a $1 \mathrm{D}$ integral is required (3), whereas the Compton spectrum involves a $2 \mathrm{D}$ integral, which will generally make it harder to discern spectral features of $\varrho(p)$ in the observed Compton profiles. On the whole, though, it would be sensible to view these two spectroscopies as being complementary, especially in light of the recent improvements in Compton techniques [24]. A note should also be made of the potential of other spectroscopies in investigating momentum densities [25].

\section{2 D ACAR and the New Superconductors; an Overview}

At present, 2D-ACAR studies are available for the superconductors $\mathrm{YBa}_{2} \mathrm{Cu}_{3} \mathrm{O}_{7-x}, \mathrm{Bi}_{2} \mathrm{Sr}_{2} \mathrm{CaCu}_{2} \mathrm{O}_{8+x}$, and $\mathrm{La}_{2-x} \mathrm{Sr}_{x} \mathrm{CuO}_{4}$, and the results are considered briefly in the following.

The $\mathrm{YBa}_{2} \mathrm{Cu}_{3} \mathrm{O}_{7-x}$ compounds, often perceived as the archetype of the new superconductors, are by far the most extensively studied ones. The early positron studies of $\mathrm{YBa}_{2} \mathrm{Cu}_{3} \mathrm{O}_{7-x}$ all involved twinned specimens. The first $2 \mathrm{D}$-ACAR results were reported by the 
Geneva group for projections in the $a-b$ plane [26], and from Argonne for the $c$-projection [27]; the latter projection has since been used in most studies owing to its suitability in investigating the Fermiology of layered compounds. The initial interpretation of these $2 \mathrm{D}$-ACAR data in terms of the existence of the Fermi surfaces gave way to a period of lull in the field as subsequent experiments failed to confirm these conclusions convincingly, and thus a consensus concerning a Fermi-surface interpretation of the 2D-ACAR data did not emerge in the positron community [28, 29]. A comprehensive overview of the work on twinned samples has been given by Barbiellini et al. [22].

The recent announcement of the $2 \mathrm{D}$-ACAR results from untwinned samples by the Texas-Livermore effort [30], the Argonne-Northeastern effort [31], and the preliminary results by the Geneva group [32] marks a significant advance in the field. It is important that the overall experimental results and their interpretation, by three independent groups, are in agreement, thereby establishing a consensus in the positron community about the electronic structure of this material. Also, a comparison between the metallic and insulating phases of $\mathrm{YBa}_{2} \mathrm{Cu}_{3} \mathrm{O}_{7-x}$ is given in [33].

On the theoretical side, band theory based calculations of the electron-positron momentum density are providing quantitative predictions that have been compared in detail with experiment. The first theoretical electron-positron momentum densities in the high- $T_{\mathrm{c}}$ 's were reported by Bansil et al. [15] and have been followed by a series of theoretical papers by these and other authors [16-20,22]. We return to the question of comparisons between theory and experiment in $\mathrm{YBa}_{2} \mathrm{Cu}_{3} \mathrm{O}_{7-x}$ in Sect. IV below.

Turning to the $\mathrm{Bi}_{2} \mathrm{Sr}_{2} \mathrm{CaCu}_{2} \mathrm{O}_{8+x}$ superconductor, two measurements have recently become available $[34,35]$, yielding very similar results. The spectra were measured in the $c$-axis projection and, for the ideal body-centered tetragonal lattice, should therefore possess $\mathrm{C}_{4 \mathrm{v}}$ symmetry (i.e. 8-fold symmetry in the plane); however, the lower $C_{2 v}$ symmetry (i.e. 4-fold symmetry in the plane) was observed in both cases. This discrepancy has been associated with the lattice modulation along the $b$-direction, caused by an insertion of extra oxygen atoms in the $\mathrm{BiO}$ layers with a period of about 5 lattice distances [36]. One then obtains an effective Brillouin zone that is smaller than the ideal zone in the $b$-direction, leading to a reduced symmetry of the spectra [34]. The presence of a sub- stantial modulation complicates the interpretation of the data, even though the limited comparison between band theory and experiment carried out so far show some agreement [35].

Finally, the $\mathrm{La}_{2} \mathrm{CuO}_{4}$ [37] 2D-ACAR results were among the first to be reported, but the interpretation seems unclear. In [38], $\mathrm{La}_{2} \mathrm{CuO}_{4}$ is studied in various projections as a function of temperature. A large isotropic distribution $(\sim 85 \%)$ was attributed to corelike electrons, and the remaining anisotropic distribution was attributed to valence electrons. No Fermi surface structures could be found in this insulating phase, as expected. The anisotropic parts of the measured spectra were compared to calculations based on an LCAO-MO model [39]. In contrast to the band theory based approach, the LCAO-type schemes involve many free parameters, but may be useful in understanding the overall shapes of the 2D-ACAR spectra in some cases.

\section{Comparison Between Theory and Experiment for $\mathrm{YBa}_{2} \mathrm{Cu}_{3} \mathrm{O}_{7}$}

We compare the measured 2D-ACAR spectra for the $c$-axis projection from an untwinned single crystal of $\mathrm{YBa}_{2} \mathrm{Cu}_{3} \mathrm{O}_{6.9}$ with the corresponding band theory predictions with reference to Figures 1-5.

Figure 1 shows that the 2D-ACAR spectrum is quite featureless. This is expected since most of the signal is associated with completely filled electron bands, with only about $3 \%$ of the contribution arising from the partially filled bands. Figure 2, which gives two representative sections through the distribution of Fig. 1, indicates that the overall shape of the asmeasured experimental spectrum differs significantly from the band theory predictions; similar discrepancies have been found to exist for twinned $\mathrm{YBa}_{2} \mathrm{Cu}_{3} \mathrm{O}_{6.9}$ samples. These discrepancies appear to arise mainly from the presence of a background in the experiment related to annihilations from various sorts of defected regions in the sample. It was established early [16], however, that if an isotropic background is substracted from the data, the measured and computed anisotropies agree in many fine details even though the overall amplitude of the experimental anisotropy is about a factor of two smaller than in the calculations.

Figure 2 also shows that the 2D-ACAR spectra in $\mathrm{YBa}_{2} \mathrm{Cu}_{3} \mathrm{O}_{6.9}$ possess a significant temperature dependence. A similar temperature effect is seen in 


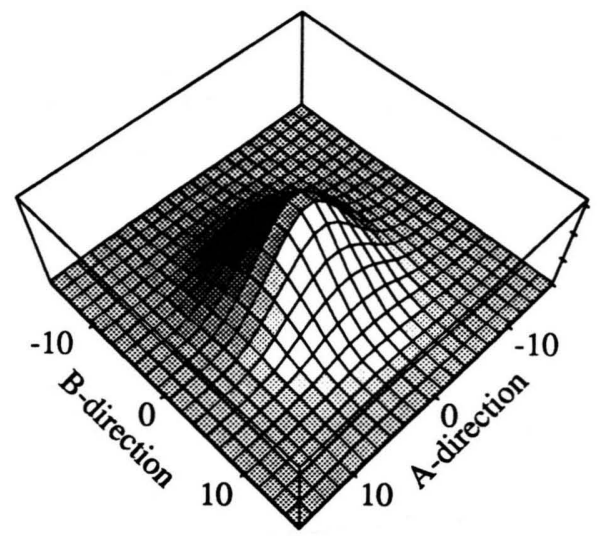

Fig. 1. The as-observed 2D-ACAR spectrum for an untwinned $\mathrm{YBA}_{2} \mathrm{Cu}_{3} \mathrm{O}_{6.9}$ sample at $30 \mathrm{~K}$.

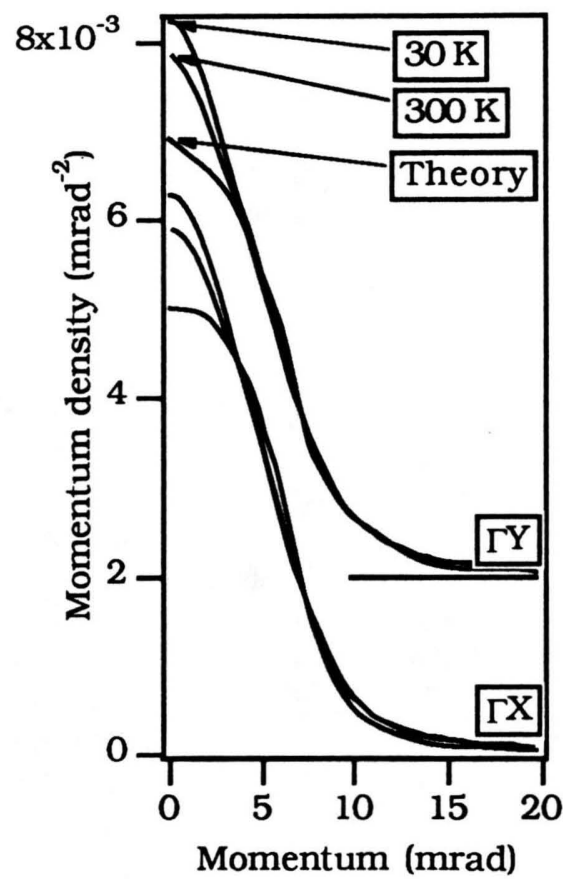

Fig. 2. Sections along $\Gamma Y$ and $\Gamma X$ through the $2 D-A C A R$ distribution in Fig. 1, together with the corresponding theoretical predictions. The data at $30 \mathrm{~K}$ as well as $300 \mathrm{~K}$ are shown.

twinned samples as well as in polycrystals [40-42]. It appears, however, that at higher temperature $(300 \mathrm{~K})$ the agreement beetween theory and experiment is somewhat better; recently, we have explored the possibility that the discrepancy between the theory and experiment may in this sense be related to the temperature effect seen in Fig. 2 [43]. An analysis of the 2D-

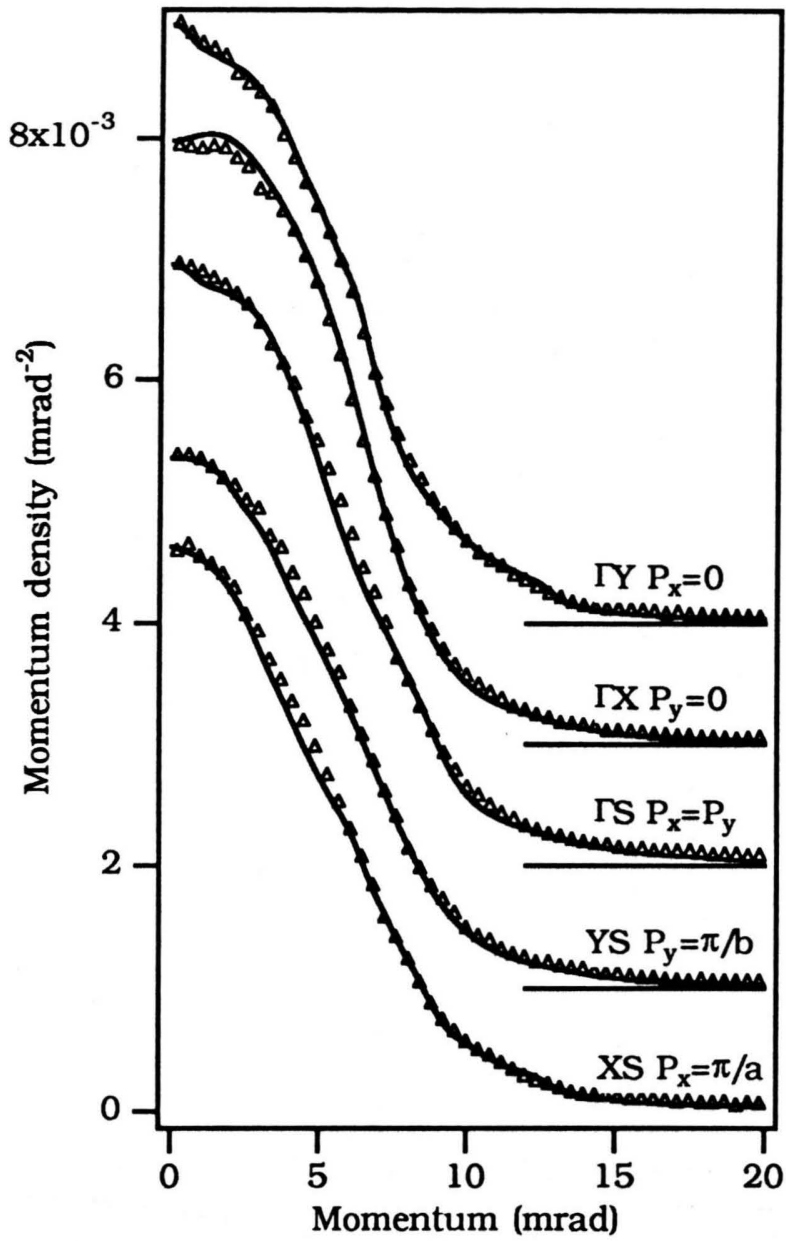

Fig. 3. A comparison of various sections through the background-corrected 2D-ACAR spectrum [43] with the corresponding theoretical predictions [16]. The results for the high-symmetry lines $\Gamma \mathrm{Y}, \Gamma \mathrm{X}, \Gamma \mathrm{S}, \mathrm{YS}$, and $\mathrm{XS}$ are shown as marked.

ACAR spectra obtained at five different temperatures, ranging from $30 \mathrm{~K}$ to $300 \mathrm{~K}$, indicated that any of the observed spectra, $M(p, T)$, could be written to a good approximation as a superposition of two temperatureindependent spectra $F(p)$ and $B(p)$ :

$$
M(p, T)=(1-I(T)) F(p)+I(T) B(p) .
$$

Here, $I(T)$ is a temperature-dependent weight factor. Equation (5) is well known from positron defect studies where it normally implies positron trapping, with $I(T)$ giving the trapping probability. By exploiting the form of (5), while using one adjustable parameter, it is possible to extract a background-corrected experimental spectrum $H(p)$, shown in Figure 3. A comparison of 

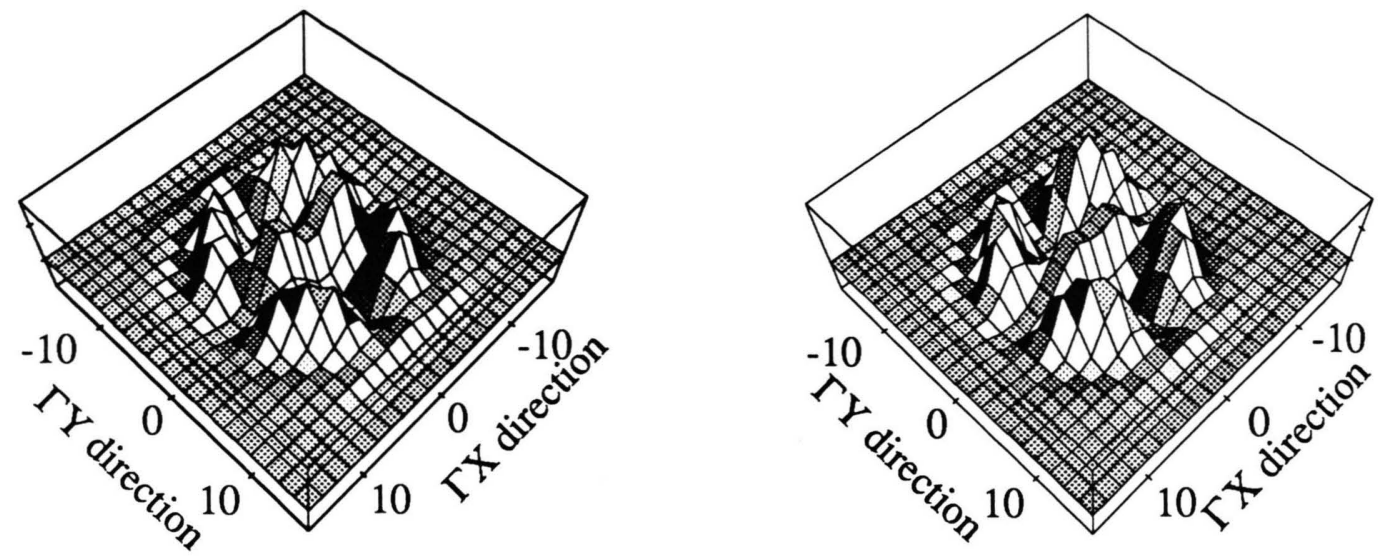

Fig. 4. Anisotropic spectra obtained by subtracting a smooth function from the as-observed data in untwinned $\mathrm{YBa}_{2} \mathrm{Cu}{ }_{3} \mathrm{O}_{6.9}$. Left frame is for measurements at $300 \mathrm{~K}$, while the right frame is for $30 \mathrm{~K}$.
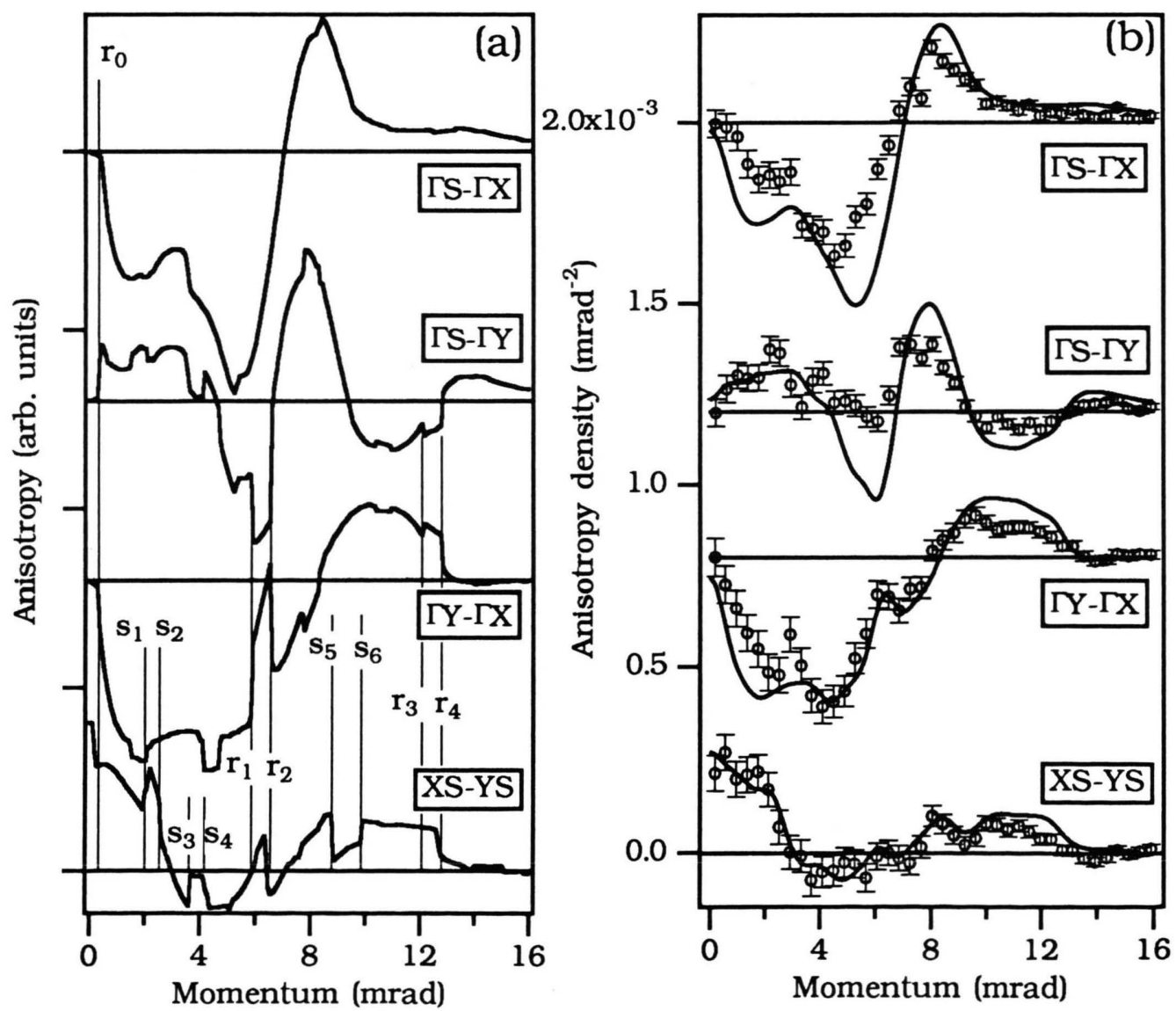

Fig. 5. (a) Theoretical anisotropic momentum distribution, here defined by taking differences between spectra along four different pairs of directions [16]. Thin vertical lines mark the position of various FS features $r_{0}, s_{1}$, etc. discussed in the text. (b) A comparison between the resolution-broadened theory curves in (a) with the corresponding experimental difference sections obtained from the corrected data [43]. The spectra have been offset relative to one another as indicated by the horizontal lines. 
Figs. 2 and 3 reveals a dramatically improved agreement between theory and experiment. Notably, in contrast to Fig. 2, the predicted anisotropy near $\boldsymbol{p}=\mathbf{0}$ is clearly manifest in the experimental data of Figure 3.

We turn now to discuss details of structure in the data by considering anisotropies of the spectra obtained by subtracting a smooth isotropic function from the spectrum of Figure 1. The result, shown in Fig. 4, is seen to be quite similar at $30 \mathrm{~K}$ and $300 \mathrm{~K}$, and consists of a central ridge-like feature surrounded by four "mountains". Additionally, a less prominent sideridge along the $\Gamma \mathrm{X}$-direction is visible at both temperatures around $12 \mathrm{mrad}$. Some temperature dependence is also apparent, e.g. the central ridge possesses two peaks whose amplitude decreases with decreasing temperature. The central ridge and the sideridge can be shown to arise mainly from the onedimensional $\mathrm{Cu}-\mathrm{O}$ chain bands and the associated Fermi surfaces (FS) running parallel to the $\Gamma \mathrm{X}$-direction, and these features possess $\mathrm{C}_{2 \mathrm{v}}$-symmetry. In contrast, the aforementioned mountains possess $\mathrm{C}_{4 \mathrm{v}}$-symmetry and may contain contributions both from the $\mathrm{Cu}-\mathrm{O}$ planes and the chains.

In order to elucidate the Fermiology of $\mathrm{YBa}_{2} \mathrm{Cu}_{3} \mathrm{O}_{7}$, in the following we frequently refer to the four FSsheets $[44,45,15]$ predicted by band theory, namely [46]: (1) a pair of closely-spaced S-centered hole sheets that possess the shape of the inner and outer surfaces of a "barrel", (2) an electron "ridge" surface running along $\Gamma \mathrm{X}$, and (3) a "pillbox" hole sheet centered at S along with an associated hole sheet mainly centered on the ГY-direction. Most calculations agree well with regard to the "barrels" and the "ridge". Some relatively minor differences are, however, found with regard to the "pillbox" and the associated hole sheets; this is expected since these sheets arise from a heavymass band that lies close to the Fermi level and are therefore sensitive to computational details.

In Fig. 5 representative anisotropic sections are shown for the background-corrected data in the form of differences along four pairs of directions. We comment on the theoretical results of Fig. $5 \mathrm{a}$ first. The complexity of these spectra arises not only from the presence of various FS sheets in $\mathrm{YBa}_{2} \mathrm{Cu}_{3} \mathrm{O}_{7}$, but also from the detailed character of the electronic wave functions of various filled bands. Several of the "breaks" associated with the Fermi surface are indicated (e.g., $r_{1}, r_{2}, s_{1}, \ldots$ etc.). The sharpness of such breaks reflects the nearly dispersionless nature of the band structure of $\mathrm{YBa}_{2} \mathrm{Cu}_{3} \mathrm{O}_{7}$ along the $c$-axis and emphasizes the advantage of using the $c$-axis projection in exploring the Fermiology of layered materials. Note that the FS breaks in Fig. 5 a occur in a distribution that itself possesses substantial underlying structure.

The signatures of various FS sheets in Fig. $5 \mathrm{a}$ are as follows. The ridge FS $\left(r_{0}\right)$ gives the rapid variation near $p=0$; since the ridge lies along the $\Gamma \mathrm{X}$-direction, in the $\Gamma \mathrm{S}-\Gamma \mathrm{X}$ spectrum or other difference spectra involving the $\Gamma$-point, the anisotropy is nearly zero for momenta (around $p=0$ ) of the order of the width of the ridge. The first umklapp of the ridge $\left(r_{1}, r_{2}\right)$ is seen clearly around $6.3 \mathrm{mrad}$ as a peak in $\Gamma \mathrm{Y}-\Gamma \mathrm{X}$, and a dip in $\Gamma S-\Gamma Y$; only weak features are seen at this momentum in the $\Gamma \mathrm{S}-\Gamma \mathrm{X}$ or $\mathrm{XS}-\mathrm{YS}$ curves. The second image of the ridge $\left(r_{3}, r_{4}\right)$ occurs around $13 \mathrm{mrad}$. The high-momentum end $r_{4}$ is generally more clearly visible than the low-momentum feature $r_{3} ; r_{4}$ in particular coincides with the zero crossing in $\Gamma S-\Gamma Y$. The pillbox FS yields relatively prominent features only in the XS-YS spectrum. The breaks $s_{1}$, $s_{2}, s_{3}$, and $s_{4}$ are all associated with the pillbox. Since the dimensions of the pillbox in the XS and YS directions are different, but the XS-YS difference must obviously vanish at the S-point (around $3.1 \mathrm{mrad}$ ), we get two breaks on each side of the S-point in the XS - YS spectrum. Interestingly, though, the image of the pillbox around $9.5 \mathrm{mrad}\left(s_{5}, s_{6}\right)$ only consists of two prominent breaks. This results from the fact that in this momentum region, the pillbox possesses a substantial signature only along the YS and not the $\mathrm{XS}$ direction. Finally, as expected, the $\mathrm{Cu}-\mathrm{O}$ plane sheets give essentially no discernible signatures in the $2 \mathrm{D}$-ACARs as a result of the weak coupling of the positron Bloch state with the $\mathrm{Cu}-\mathrm{O}$ planes [15-20]. Figure $5 \mathrm{~b}$ shows that experimental anisotropies are in good accord with theory with regard to the details of the spectral features. Also there is good agreement concerning the absolute amplitude of the undulations between theory and experiment. The level of discrepancies seen in Fig. 5 b is common in even simpler materials in first-principles comparisons between theory and experiment.

FS signatures in the experimental spectra can now be elucidated with reference to Figure $5 \mathrm{~b}$. We consider the ridge FS first. As noted above, the ridge FS gives three distinct features: (1) rapid variation in anisotropy near $p=0,(2)$ an image around $6.3 \mathrm{mrad}$, and (3) a second image around $13 \mathrm{mrad}$. The experimental spectra display all three of these features. Although we 
do not, of course, expect any "breaks" in the experimental data owing to resolution effects, near $p=0$ both sets of curves in Fig. $5 \mathrm{~b}$ possess similarly rapid variations and slopes; the width of the experimental ridge would, however, appear to be somewhat larger than that implicit in our band structure. The $6.3 \mathrm{mrad}$ image of the ridge $\left(r_{1}, r_{2}\right)$ is particularly important in the $\Gamma Y-\Gamma X$ curve, because this feature lies on a relatively gently varying background and remains quite prominent in the theory even after including resolution broadening, and it is crucial that this feature is indeed observed clearly in the experimental data. By contrast, in the $\Gamma \mathrm{S}-\Gamma \mathrm{Y}$ curve, the resolution broadening effects would make the $6.3 \mathrm{mrad}$ ridge image difficult to distinguish from the background. Finally, the image of the ridge FS near $13 \mathrm{mrad}\left(r_{3}, r_{4}\right)$ becomes rather smooth in theory upon resolution broadening. We emphasize that the shape of the experimental data in this momentum range is quite consistent with the theory. A closer examination of this region shows that the experimental edge around $13 \mathrm{mrad}$ is just about as sharp as that predicted theoretically. Also, in the case of $\Gamma \mathrm{S}-\Gamma \mathrm{Y}$, both theory and experiment show the related zero crossing around $13 \mathrm{mrad}$.

Concerning the pillbox, as noted, the prominent features involve the XS-YS anisotropic spectrum, where the theory and experiment appear to be in accord in Fig. 5 b, although upon resolution broadening the theoretical breaks $s_{1}$ through $s_{4}$ become rather indistinct and are difficult to identify in the experimental spectra. The dip arising from the breaks $s_{5}$ and $s_{6}$ remains distinct even after resolution effects are included, and in this momentum region the experimental data display a similar dip in accord with the theory. Further analysis of the 2D-ACAR data is nevertheless needed to establish the existence of the pillbox FS firmly via the present experiments. Finally, it may be noted that the present results are complementary to the dHvA and angle-resolved photoemission results concerning the Fermi surface features [47-49].

[1] H. Kamerlingh Onnes, Akad. van Wetenschappen, Amsterdam 14, 113, 818 (1911).

[2] J. Bardeen, L. N. Cooper, and J. R. Schrieffer, Phys. Rev. 106, 162 (1957); 108, 1175 (1957).

[3] G. Bednorz and K. A. Müller, Z. Physik B 64, 189 (1986).

[4] For a recent discussion of these topics see, e.g., Proc. of the Argonne Fermiology Workshop, J. Phys. Chem. Solids 52 (1991).

\section{Conclusions}

We have presented an overview of the presently available $2 \mathrm{D}$-ACAR positron annihilation results for the high- $T_{\mathrm{c}}$ superconductors, with focus on $\mathrm{YBa}_{2} \mathrm{Cu}_{3} \mathrm{O}_{7-x}$, which has been investigated most intensely. The observed spectra are shown to be in excellent accord with the predictions of the local density approximation (LDA) based on band theory framework, some complications caused by the presence of a background of an uncertain origin notwithstanding. The crucial role played by theoretical computations in interpreting the observations in the high- $T_{\mathrm{c}}$ 's indicates that progress in exploring the Fermiology of complex materials via the positron technique in the future will require close co-operation between theory and experiment.

The present positron annihilation results concerning the Fermi surface of $\mathrm{YBa}_{2} \mathrm{Cu}_{3} \mathrm{O}_{7}$, when combined with complementary results of angle-resolved photoemission and dHvA experiments, leave little doubt that the Fermiology of the normal-state $\mathrm{YBa}_{2} \mathrm{Cu}_{3} \mathrm{O}_{7}$ is described quite reasonably by the conventional band-theory based picture of the metallic state. These results provide significant experimental constraints on the physically relevant theoretical models of the Fermi gas in the high- $T_{\mathrm{c}}$ 's, even though these observations do not by themselves reveal the superconducting mechanism at work in these materials.

\section{Acknowledgements}

The present work was supported by the U.S. Department of Energy, Basic Energy Sciences, Division of Material Sciences under contract \#W-31-109ENG-38 (LS, AB), including a subcontract to Northeastern University (AB), and a NATO travel grant. This work benefited from the allocation of supercomputer time on the NERSC and Pittsburgh Supercomputer centres.

[5] R. Beringer and C. G. Montgomery, Phys. Rev. 61, 222 (1942).

[6] S. De Benedetti, C. E. Cowan, W. R. Konneker, and H. Primakoff, Phys. Rev. 77, 205 (1950).

[7] G. Lang and S. De Benedetti, Phys. Rev. 108, 914 (1957).

[8] A. T. Stewart, Can. J. Phys. 35, 168 (1957).

[9] S. Berko and J. S. Plaskett, Phys. Rev. 112, 1877 (1958).

[10] S. Berko, M. Haghgooie, and J. J. Mader, Phys. Lett. A 63, 335 (1977). 
[11] A. A. Manuel, S. Samoilov, Ø. Fischer, and M. Peter, Helv. Phys. Acta 52, 255 (1979).

[12] R. N. West, J. Mayers, and P. A. Walters, J. Phys. E 14, 478 (1981).

[13] S. Berko, in: Momentum Distributions (R. N. Silver and P. E. Sokol, eds.), Plenum Press, New York 1989, p. 273. - P. E. Mijnarends, in: Positron Solid State Physics, Course LXXXIII, Int. School of Phys. "Enrico Fermi" (W. Brandt and A. Dupasquier, eds.), North-Holland, Amsterdam 1983, p. 146.

[14] See e.g., B. Williams (ed.), Compton Scattering, McGrawHill, London 1977; see also M. J. Cooper, Rep. Prog. Phys. 48, 415 (1985).

[15] A. Bansil, R. Pankaluoto, R. S. Rao, P. E. Mijnarends, W. Dlugosz, R. Prasad, and L. C. Smedskjaer, Phys. Rev. Lett. 61, 2480 (1988).

[16] A. Bansil, P. E. Mijnarends, and L. C. Smedskjaer, Physica C 172, 175 (1990). - A. Bansil, J. Phys. Chem. Solids 52, 1493 (1991).

[17] A. Bansil, P. E. Mijnarends, and L. C. Smedskjaer, Phys. Rev. B 43, 3667 (1991).

[18] S. Massidda, Physica C 169, 137 (1990).

[19] D. Singh, W. E. Pickett, E. C. von Stetten, and S. Berko, Phys. Rev. B 42, 2696 (1990).

[20] T. Jarlborg, B. Barbiellini, E. Boronski, P. Genoud, and M. Peter, J. Phys. Chem. Solids 52, 1515 (1991).

[21] P. E. Mijnarends and A. Bansil, J. Phys.: Cond. Matter 2, 911 (1990)

[22] B. Barbiellini, P. Genoud, J. Y. Henry, L. Hoffmann, T. Jarlborg, A. A. Manuel, S. Massidda, M. Peter, W. Sadowski, H. J. Scheel, A. Shukla, A. K. Singh, and E. Walker, Phys. Rev. B 43, 7810 (1991).

[23] For recent reviews see: J. Arponen and E. Pajanne, J. Phys. F 9, 2359 (1979). - H. Sormann and M. Sob, Phys. Rev. B 41, 10529 (1990).

[24] See e.g., this conference: M. Ito et al., N. Shiotani, N. Sakai, K. Namikawa, L. Dobrzyński; see also N. Shiotani et al., Proc. 8th Int. Conf. on Positron Annihilation (L. Dorikens-Vanpraet, M. Dorikens, and D. Segers, eds.), World Scientific 1989, p. 122.

[25] E. Weigold, Z. Naturforsch. 48a, 371 (1993). - F. Bell, Th. Tschentscher, J. R. Schneider, and A. J. Rollason, Z. Naturforsch. 48a, 273 (1993).

[26] L. Hoffmann, A. A. Manuel, M. Peter, E. Walker, and M. A. Damento, Europhys. Lett. 6, 61 (1988). - L. Hoffmann, A. A. Manuel, M. Peter, E. Walker, and M. A. Damento, Physica C 153-155, 129 (1988). - M. Peter, IBM J. Res. Dev. 33, 333 (1988).

[27] L. C. Smedskjaer, J. Z. Liu, R. Benedek, D. G. Legnini, D. J. Lam, M. D. Stahulak, H. Claus, and A. Bansil, Physica C 156, 269 (1988).

[28] M. Peter and A. A. Manuel, Phys. Scripta T 29, 106 (1989).

[29] H. Haghighi, J. H. Kaiser, S. Rayner, R. N. West, M. J. Fluss, R. H. Howell, P. E. A. Turchi, A. L. Wachs, Y. C. Jean, and Z. Z. Wang, J. Phys. Cond. Matter 2, 1911 (1990).

[30] H. Haghighi, J. H. Kaiser, S. Rayner, R. N. West, J. Z. Liu, R. Shelton, R. H. Howell, F. Solar, and M. J. Fluss, Phys. Rev. Lett. 67, 382 (1991); J. Phys. Chem. Solids 52, 1535 (1991).
[31] L. C. Smedskjaer, A. Bansil, U. Welp, Y. Fang, and K. G. Baily, J. Phys. Chem. Solids 52, 1541 (1991).

[32] L. Hoffmann, W. Sadowski, and M. Peter, Proceedings of the 9th Int. Conf. on Positron Annihilation, Hungary 1991.

[33] M. Peter, T. Jarlborg, A. A. Manuel, B. Barbiellini, and S. E. Barnes, Z. Naturforsch. 48a, 390 (1993).

[34] P. E. Mijnarends, A. F. J. Melis, A. W. Weber, A. A. Menovshy, and K. Kadowaki, Physica C 176, 113 (1991).

[35] L. P. Chan, D. R. Harshman, K. G. Lynn, S. Massidda, and D. B. Mitzi, Phys. Rev. Lett. 67, 1350 (1991).

[36] Y. Le Page, W. R. McKinnon, J. M. Tarascon, and P. Barboux, Phys. Rev. B 40, 6810 (1989). - E. A. Hewat, J. J. Capponi, and M. Marezio, Physica C 157, 502 (1989). - A. Yamamoto, M. Onoda, E. Takayami-Muromachi, F. Izumi, T. Ishigaki, and H. Asano, Phys. Rev. B 42, 4228 (1990). - M. D. Kirk, J. Nogami, A. A. Baski, D. B. Mitzi, A. Kapitulnik, T. H. Geballe, and C. F. Quate, Science 242, 1673 (1988).

[37] S. Tanigawa, Y. Mizuhara, Y. Hidaka, M. Oda, M. Suzuki, and T. Murakami, Mater. Res. Soc. 5, 57 (1988).

[38] P. E. A. Turchi, A. L. Wachs, K. H. Wetzler, J. H. Kaiser, R. N. West, Y. C. Jean, R. H. Howell, and M. J. Fluss, J. Phys. Cond. Matter 2, 1635 (1990).

[39] T. Chiba, J. Chem. Phys. 64, 1182 (1976).

[40] L. Hoffmann, W. Sadowski, A. Shukla, Gh. Adam, B. Barbiellini, and M. Peter, J. Phys. Chem. Solids 52, 1551 (1991).

[41] L. C. Smedskjaer, B. W. Veal, D. G. Legnini, A. P. Paulikas, and L. J. Nowicki, Phys. Rev. B 37, 2330 (1988); Physica B 150, 56 (1988)

[42] E. C. von Stetten, S. Berko, X. S. Li, R. R. Lee, J. Brynestad, D. Singh, H. Krakauer, W. E. Pickett, and R. E. Cohen, Phys. Rev. Lett. 60, 2198 (1988).

[43] L. C. Smedskjaer, A. Bansil, U. Welp, Y. Fang, and K. G. Bailey, Physica C 192, 259 (1992). - L. C. Smedskjaer and A. Bansil, Proceedings of the 9th Int. Conf. on Positron Annihilation, Hungary 1991.

[44] J. Yu, S. Massidda, A. J. Freeman, and D. D. Koelling, Phys. Lett. A 122, 203 (1987).

[45] W. E. Pickett, R. E. Cohen, and H. Krakauer, Phys. Rev. B 42, 8764 (1990).

[46] The nomenclature for the basal plane of the Brillouin zone is commonly given as: $\Gamma$ is the centre of the zone, $\mathrm{X}(\mathrm{Y})$ is the point where the $x$-axis ( $y$-axis) intercepts the zone boundary, $S$ is the corner point of the zone. The crystal $a$-direction is parallel to the $x$-axis, $b$ to the $y$-axis.

[47] J. C. Campuzano, L. C. Smedskjaer, R. Benedek, G. Jennings, and A. Bansil, Phys. Rev. B 43, 2788 (1991).

[48] C. M. Fowler, B. L. Freeman, W. L. Hults, J. C. King, F. M. Mueller, and J. L. Smith, Phys. Rev. Lett. 68, 534 (1992). - F. M. Mueller, J. Phys. Chem. Solids 52, 1457 (1991). - J. L. Smith, C. M. Fowler, B. L. Freeman, W. L. Hults, J. C. King, and F. M. Mueller, to appear in Proc. of Third Int. Symp. on Superconductivity, Sendai, Japan.

[49] G. Kido, K. Komorita, H. Katayama-Yoshida, and T. Takahashi, J. Phys. Chem. Solids 52, 1465 (1991). 